\title{
Design and implementation of power locking and release functions in China power market trading activity
}

\author{
Ping Shao ${ }^{1, a,{ }^{*}}$, Gaoqin Wang ${ }^{1, b}$, Yanmin Guo ${ }^{1, \mathrm{c}}$, Wenzhe Zhang ${ }^{2, \mathrm{~d}}$, Guoning \\ $\mathrm{GaO}^{3, \mathrm{e}}$ \\ ${ }^{1}$ China Electric Power Research Institute, Nanjing 210003, China \\ ${ }^{2}$ Chongqing Electric Power Grid Co., Ltd, Chongqing 410014,China \\ ${ }^{3}$ Zhejiang Electric Power Grid Co., Ltd, Hangzhou 310007,China
}

ashaoping@epri.sgcc.com.cn, bwanggaoqin@epri.sgcc.com.cn, ${ }^{\mathrm{c}}$ guoyanmin@epri.sgcc.com.cn, ${ }^{d}$ zwz@cq.sgcc.com.cn, ${ }^{e}$ gao_guoning@zj.sgcc.com.cn

\begin{abstract}
With the adjustment of China's National Energy Policy, all kinds of power market trading activity carried out, because some plants too much involved in trading activities that far exceeds its maximum generation capacity, most likely caused by not complete all transactions of electricity. This paper presents a method for generating business transactions can be locked and release electricity, through the real-time monitoring service in the background, when the power generation enterprises in concurrent transaction process, the program can automatically lock has been declared the electricity trading. Meanwhile, after clearing the transaction, the program automatically releases the locked transaction electricity, and by checking the largest power generation company's own generating capacity, limiting its participation in excessive trading activity that is far beyond the scope of their power generation capacity. Designed by this method function modules have been good engineering applications.
\end{abstract}

Keywords: Power market, Transaction, Power lock, Power release.

\section{适用中国电力市场并发交易活动中电量锁定与释放的功能设计与实现}

\author{
邵平 ${ }^{1, a,{ }^{*}}$, 王高琴 ${ }^{1, b}$, 郭艳敏 ${ }^{1, c}$, 张文哲 ${ }^{2, d}$, 高国宁 ${ }^{3, e}$ \\ ${ }^{1}$ 中国电力科学研究院, 鼓楼区, 南京市, 江苏省, 中国 \\ ${ }^{2}$ 国网重庆电力公司, 渝中区, 重庆市, 中国 \\ ${ }^{3}$ 国网浙江电力公司, 西湖区, 杭州市, 浙江省, 中国 \\ ashaoping@epri.sgcc.com.cn, bwanggaoqin@epri.sgcc.com.cn, gooyanmin@epri.sgcc.com.cn, \\ dzwz@cq.sgcc.com.cn, ${ }^{\mathrm{e}}$ gao_guoning@zj.sgcc.com.cn
}

中文摘要. 随着国家能源政策的调整, 各类 电力市场化交易活跃开展, 发电企业因参与 过多的交易, 以至于超过自身发电能力, 有
可能造成后期无法履行交易情况。本文提出 了发电企业交易电量锁定和释放的概念, 通 过后台服务实时监视，实现了对发电企业在 参加并发交易时, 自动锁定已经申报的交易 
电量, 同时在交易出清后自动释放被锁定的 交易电量, 并通过校验发电企业市场成员的 最大发电能力, 限制发电企业参与超出自身 发电能力范围交易的方法。通过该方法设计 的功能模块在电力市场交易运营技术支撑 平台上得到良好的工程应用, 确保发电企业 具备执行已经签订的交易电量合约的能力。

\section{关键词: 电力市场;发电企业; 交易; 电量; 锁} 定;释放

\section{1. 引言}

随着中国国家能源政策的调整, 各类市 场化的电力交易不断开展, 特别是2013年国 家能源局为了推进电力用户与发电企业直 接交易 [1-2], 试点推广工作由各地根据实际 情况继续推进电力用户与发电企业直接交 易相关工作。发电企业可以参与市场各类交 易品种在不断增加, 除电力用户直接交易 外, 还有如外送电[3-4]、发电权交易 [5-7] 等, 以及发电企业自身交易策略[8-9]的调 整, 发电企业的交易电量在总发电量的占比 不断的提高。而随之带来的问题是发电企业 市场成员可以参加多个市场的并发交易, 而 由于各交易的时间周期[10]的不同，并未能 完成最终的交易过程, 所以无法限制发电市 场成员参与, 很可能造成交易能力远远超出 发电企业的最高发电能力。因此, 如何通过 新的技术方法和手段限制发电市场成员过 多的参与超出自身发电能力的交易带来了 困扰。

本文介绍了一种电力交易模块 [11]的 设计方法, 实现了对发电企业在参加并发交 易时, 自动锁定已经申报的交易电量, 同时 在交易出清后自动释放被锁定的交易电量, 并通过校验发电企业市场成员的最大发电 能力, 限制发电企业参与超出自身发电能力 范围的交易, 确保发电企业具备执行已经签 订的交易电量合约[12-13]的能力, 通过规则 完成所电能量的结算[14-15]。

\section{2. 业务现状分析}

电力市场交易是买卖双方对电力产品 进行磋商谈判的一种生意, 交易过程是电网
公司与其他交易主体就买卖电能进行硡商 谈判的流程。目前, 国内已经开展了多个周 期类型的电力市场交易，如图1所示，包括 年、季、月以及短期的多种周期的交易。与 国外成熟电力市场交易不同, 国外的电能量 交易以电力为约束, 而国内主要是以电量为 约束的交易, 同时这些交易的结果都需要根 据结算的要求将电量分解到月。

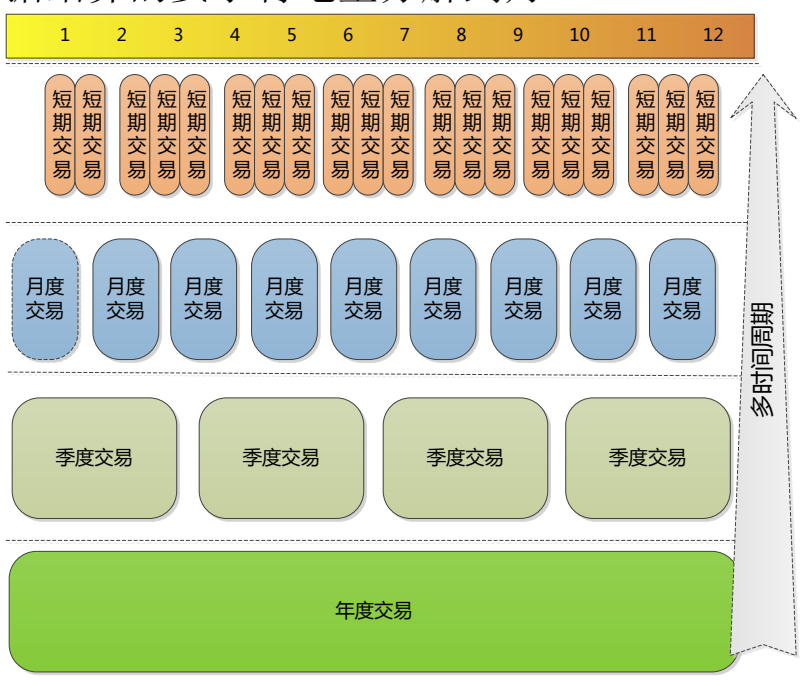

图1-电力市场多种交易周期

当前, 中国国内的电力市场交易以国外 最大的区别是, 国内的市场交易和结算都以 物理电量为主, 而国外市场[16-17]开展通常 是金融期货和现货交易，以及双结算的方 式。因此, 国内市场成员在参与电力市场交 易过程中的交易电量受最大可发电能力的 限制, 也就是说发电企业会受自身设备的检 修、停备, 以及线路以及输变电设备的检修 的影响。由于国内的市场并未真正的开展, 交易业务活动通常每年会组织开展可数的 几次，且并发交易活动也并不多见。而传统 的交易系统中虽然在默认申报时不允许超 过最大的发电能力, 但对申报电量约束的计 算仅限于对市场交易出清结果后总加与剩 余可用发电量之和。

随着国内电力市场改革的不断推进, 电 力市场交易活动越来越频繁，如图2所示, 发电企业也可参与多个并发的市场交易业 务, 交易中心在传统的系统中无法对其交易 申报电量做并发控制, 在并发交易出清后极 有可能的远远超出发电企业的最大发电能 力。同时, 国内也在考虑允许发电企业申报 超出给定限额范围内的电量, 超出的部分可 
以通过发电权替代等方式进行交易。虽然国 内在电力市场交易信息化支撑方面能力取 得了长足进步, 但与现有的业务需求存在一 定的差距。所以, 需要一种可以指定申报超 出的最大可用发电能力的限额比例, 且支持 并发交易过程的交易管理模块, 支撑现有的 电力市场并发交易业务的开展。

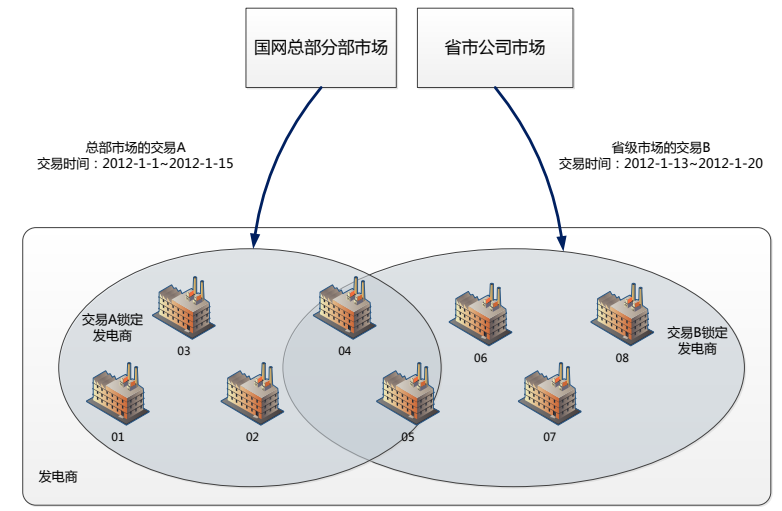

图2 多个市场的并发交易

\section{3. 功能设计}

交易管理是对电力商品或服务的买卖 活动进行的全过程管理, 本次提出的功能主 要是支持申报电量锁定与释放交易管理部 分的功能设计, 其业务流程如图3所示, 主 要包括交易序列发布、交易申报、检验有效 发电能力、交易电量锁定、交易电量释放几 部分:

\section{（1）交易序列发布}

交易序列是贯穿整个交易流程的重要 标识, 交易序列发布则是组织一笔交易的第 一步。

交易序列主要描述交易的详细信息 (例) 如段数、轮次等)、定义交易的条款信息 (例 如电量规模等）、定义交易的准入规则、申 报规则、和出清规则, 定义交易的流程信息

（例如申报开始时间等，同时设置交易公 告, 设置短信提醒的内容。

对于跨区跨省双边交易, 还需要设置交 易路径。对于跨区跨省集中交易, 需要枚举 所有的买方和卖方之间的交易路径。

交易序列创建完毕, 就可以进入交易的 具体流程, 开展申报、撮合、安全校核等交 易步骤。

（2）交易申报
交易申报是电力市场交易活动中起始 的环节之一, 市场组织者根据电量买卖方的 意向组织开展交易, 预先设定准入市场成员 并同时发布交易公告, 发电企业自身的情况 按照市场交易规则和条件, 开展此次交易的 申报活动, 主要申报电量、电价等数据, 申 报的电量需要经过检验有效发电能力后才 能正式上报。

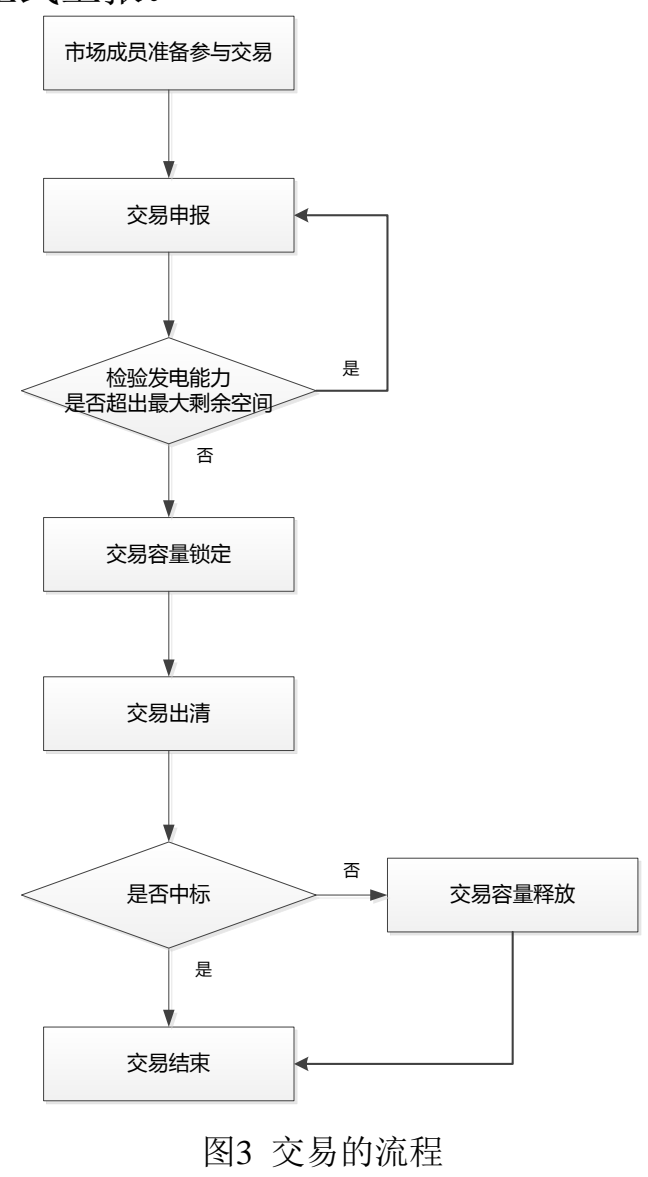

(3) 申报电量检验

申报电量检验主要是用以限制发电交 易参与者的最大可申报电量, 避免其超过自 身发电能力或者线路和断面的安全限额。这 首先得计算发电企业的剩余发电能力空间, 也就是发电企业已经投产的发电机组在交 易周期内去除已经签订交易合约电量后的 最大剩余可发电量; 其次, 发电企业可申报 的电量不仅受自身剩余发电能力空间的影 响, 还会受到其参与并发交易电量锁定空间 的影响, 简单的说即可申报的最大电量一般 不能超过剩余的可发电量与交易锁定电量 之和。

在模块上对有申报电量的计算, 是通过 对交易周期内的平衡分析和各机组的预先 安排电量计划信息 (包括已安排的基数电量 
计划、既定交易计划、检修情况及交易相关 的线路、断面检修信息), 计算参与交易的 市场成员或机组的申报电量限额。计算结果 允许人工修正，且交易申报限额的计算时间 跨度, 和交易周期一致。本设计主要考虑电 厂发电能力的上限限额, 以及发电权交易中 转让方和受让方交易电量限额的计算, 对于 购电方交易电量限额暂不予考虑。在业务流 程上对检验不通过的申报必须退回, 用户可 以重新申报, 通过后则可正式上。同时, 由 于电网安全运行管理的需要可能会留用一 定的旋转备用容量以及考虑到发电设备的 停备或检修等情况, 会在系统设计中预留发 电最大发电负荷率的参数。

（4）交易电量锁定

交易电量锁定是指在发电企业通过发 电能力的校验后, 锁定了申报的所有有效电 量。在电力市场交易运营系统中设计中考虑 采用后台服务在数据库中自动记录该企业 被锁定的交易电量, 包括锁定对象 (发电企 业）、生效时间、失效时间、锁定的电量、 状态 (置为 “锁定” 状态) 等信息, 该部分 电量将影响在该企业交易出清前的有效发 电能力空间, 通过锁定电量可以有效限定发 电企业在参加并发交易时可能超出自身最 大发电能力的情况。

\section{（5）无约束交易结果出清}

交易申报后按规定的交易规则进行出 清计算, 一般来说, 双边交易直接由交易中 心负责对无约束的交易结果进行确认操作; 而集中交易需要按电价、环保、排放等因素 进行排序, 最后由系统自动计算出交易结 果。这些都是无约束的交易结果, 必须由调 度部门进行安全校核才能发布。

（6）有约束交易结果出清(安全校核)

无约束的交易结果出清后必须通过调 度中心负责进行安全校核, 调度部门通过对 机组、线路和其它输变电设备的运行计划, 校核该批次的交易是否符合安全校核, 该校 核过程在国内都是由调度系统实现的, 安全 校核的结果支持人工调整。经过安全校核后 形成有约束的交易结果, 并向发电企业发 布。

\section{（7）交易电量释放}

有约束的交易结果出清后, 需要对未中 标的发电企业申报的交易电量进行释放, 而 已经中标的单位则根据实际出清的电量继 续锁定其电量。在交易系统设计中采用后台 调度服务, 由出清环节结束后自动触发该服 务, 服务自动将数据库中该笔交易锁定电量 的记录置为 “释放” 状态, 通过交易电量释 放可将此部分锁定的电量空间 “返还” 给发 电企业, 将不会影响发电企业参加后续的交 易申报限制。

\section{4. 实验验证}

下面将通过具体的实例说明说明交易 电量的锁定和释放过程。

假定某发电企业装机电量600MW, 最 大发电负荷率为 $95 \%$, 则该企业在 7 月份最 大发电能力为: $600 * 24 * 31 * 0.95=424,080 \mathrm{MWH}$; 同时该企 业已经签订的合约在7月份需要执行发电量 为350,000MWH, 则剩余发电能力空间为 424,080-350,000=74,080MWH。

该企业拟同时参加在 7 月执行的 2 笔月 度交易, 申报时间均为 5 月 10 日24时前, 出 清时间为 5 月 11 日 24 日前。

该企业拟申报第一笔交易的电量 $50,000 \mathrm{MWH}$ ，第二笔交易电量 $40,000 \mathrm{MWH}$ 。当5月 10 日9: 00 申报第一笔 交易后, 交易电量自动锁定 $50,000 \mathrm{MWH}$; 当天9: 10 分申报第二笔电量时, 校验有效 发电能力时限定最大允许申报电量为 $74,080-50,000=24,080 \mathrm{MWH}$, 自动提示申报 电量超过最大允许申报电量, 则该企业按 $24,080 \mathrm{MWH}$ 申报电量, 申报后自动锁定相 同交易电量。11日出清时该企业第1笔交易 未中标, 第 2 笔交易中标全部电量 $24,080 \mathrm{MWH}$, 系统自动释放未成交的 $50,000 \mathrm{MWH}$ 空间, 该企业在6月份的剩余发 电能力变为 $74,080-24,080=50,000 \mathrm{MWH}$ 。

\section{5. 结语}

交易系统可通过基于本文中提出的并 发交易下的交易电量的锁定与释放设计方 法, 实现对参加并发交易的市场成员进行申 报交易电量的锁定。该设计有如下 2 个优点: 
1、发电企业交易电量锁定和释放的概 念, 实现了发电企业在参加并发交易时, 自 动锁定已经申报的交易电量, 同时在交易出 清后自动释放被锁定的交易电量。

2、提出了检验发电企业有效发电能力 的新方法, 不仅考虑已经签订的交易合约电 量、最大负荷率等限制, 而且增加了企业参 加并发交易后被锁定的交易电量空间的影 响, 对检验发电企业的有效发电能力边界条 件进行了扩展。

通过此设计方法可在电力市场交易运 营系统中, 实现对发电企业市场成员在参与 并发交易时申报的电量进行有效检验, 限制 发电企业参与超出自身发电能力范围的交 易电量申报, 确保发电企业在交易周期内具 备执行交易合约电量的空间。

\section{致谢}

本文为国家电网公司科技项目《面向统 一运营的互联电力市场关键技术研究与应 用》(DZN17201400039)的阶段性成果之一。

\section{References}

[1]China national energy administration department to carry out "on the current electricity users and power companies deal directly related matters notice" (2013) No. 258, http://news.cnfol.com/guoneicaijing/2014 0211/16946015.shtml;

[2]Fu-bin, LIU, et al. "Pilot and Promotion Recommendations for Direct Trading between Power Users and Plants." East China Electric Power (2013).

[3]Dexiang, JIA, and BAI Jianhua. "Research on the Transmission Mode of Wind Power Bases." Energy Technology \& Economics (2011).

[4]ARi-sheng, FANG, et al. "Multi-object hierarchical optimization research of gate trading capability based on economic operation of power system." Journal of Fuzhou University (2013).

[5]ZHANG Xian,GENG Jian,PANG Bo et al.Application and Analysis of
Generation Right Trade in Energy-saving and Emission Reduction in China[J].Automation of Electric Power Systems,2014,38(17):87-90.

[6]Jincheng, SHANG, and HE Yang. "Generation Right Exchange Theory And Its Applications Based On Energy-Saving And Emission-Reducing. Part Two. Generation Right Exchange Analysis And Applications." Dianli Xitong Zidonghua/automation of Electric Power Systems 33.13(2009):37-42.

[7]Ming, ZHOU, et al. "Impacts of Generation Rights Transaction on Transmission Service Cost." Power System Technology 34.1(2010):134-138.

[8]WU Yulin1 , WEN Fushuan2 , DING Jianying3 , HONG Huihong3 , LI Chengjun3.A Preliminary Investigation on Bidding Strategies for Generation Companies in Generation-right Trading Markets[J].Automation of Electric Power Systems,2010,34(17):6-11.

[9]Hao-yong, CHEN, et al. "Research on Transaction Mode of Direct Power Purchase by Large Consumers in Electricity Market." Power System Technology 32.21(2008):85-90.

[10]Senlin, ZHANG, et al. "Successful bilateral transactions in South China electricity market." Electric Power Automation Equipment 29.9(2009):94-100.

[11]HUANG Jie1, 2 ， XUE Yusheng2, 1 , WEN Fushuan3, XU Jianbing2, XUE Feng2, Zhaoyang DONG4.A Review of Power Market Simulation Platform[J].Automation of Electric Power Systems, 2011,35(9):6-13.

[12]CHENG Haihua, YANG Zhenglin, CAO Rongzhang.The Development of Rule Library and Algorithm Library for Electricity Market Trading[J].Automation of Electric Power Systems,2010,34(3):49-52. 
[13]ZENG Ming, JIA Zhuo.A Dynamic Programming Method for Electricity Bilateral Contracts Trading Considering Renewable Energy Generation[J].Automation of Electric Power Systems,2011,35(4):29-34.

[14]ZHANG Sen-lin . Practical Research on Settlement Mode of Transacted Electricity Quantity in Regional Electricity Market. Power System Technology, 2008, 32(3): 56-61 .

[15]LONG Suyan,ZHANG Xian,YANG Zhenglin,GENG Jian and HUANG Longda.A Component Library Based
Flexible Electricity Settlement Method[J].Automation of Electric Power Systems, 2013,37(14):89-94.

[16]LASioshansi, Fereidoon P. "Competitive Electricity Markets: Questions Remain about Design, Implementation, Performance." Electricity Journal 21(2008):74-87.

[17]Fan, Zhenyu, et al. "An overview of PJM energy market design and development." International Conference on Electric Utility Deregulation and Restructuring and Power Technologies. IEEE, 2008:12 17. 